\title{
Type 1 Myringoplasty and Platelet Gel Surgical Approach: A Case Report
}

\section{Colosio ${ }^{1}$ | N. Tanzi $^{1}$ | G.N. Frau ${ }^{2}$ | A. F. Cristallo ${ }^{*}$}

*Correspondence: Attilio Fabio Cristallo

Address: ${ }^{1}$ Azienda Sanitaria per i Servizi Sanitari della Provincia Autonoma di Trento (APSS), Immunohematology and Transfusion Service, Trento, Italy; ${ }^{2}$ Azienda Sanitaria per i Servizi Sanitari della Provincia Autonoma di Trento (APSS), Unit of Otolaryngology Ospedale di Rovereto, Trento, Italy

e-mail $₫:$ attcris@bls.it

Received: 01 March 2021; Accepted: 05 March 2021

Copyright: (C) 2021 Colosio M. This is an open-access article distributed under the terms of the Creative Commons Attribution License, which permits unrestricted use, distribution, and reproduction in any medium, provided that the original work is properly cited.

\section{ABSTRACT}

Tympanoplasty is indicated to repair tympanic membrane perforations in patients affected by hearing loss. We investigated the outcomes of unconventional microscopic approach to type 1 tympanoplasty with autologous platelet gel in tympanic membrane perforation. Considering that platelet gel improves reparation of cutaneous lesions, we evaluate the efficacy of platelet gel application in patients with a damage of a particular soft tissue, as tympanic membrane (Adler and Kent, 2002; Bhanot and Alex, 2002; Mann et al., 2001; Pierce et al., 1991). We treated a patient referred to Otorinolaryngology Clinic of S. Maria del Carmine Hospital, Rovereto (TN) with a diagnosis of tympanic membrane perforation and mixed left hearing loss who undergone to a type 1 tympanoplasty with platelet gel. Our primary outcome was tympanic index closure. We report a faster recovery in this patient treated with platelet gel as an outpatients procedure.

In conclusion, platelet gel application in otosurgery, in outpatients treatment. is a noninvasive, safe technique to restore tympanic perforation. However, further prospective data are needed to confirm these preliminary results.

Keywords: Type 1 Tympanoplasty, Autologous Platelet Gel, Tympanic Index Closure

\section{Introduction}

A 45-year-old man presented with mixed left hearing loss, persistent otitis at least seven months with otalgia, fullness and discomfort. Clinical examination revealed single, inferior and marginal, "kidney shape" tympanic membrane perforation, with mild reddened and exuding margins, surrounded by an area of miringitis (Fig. 1). The patient had been previously treated for two weeks with antibiotic therapy for a positive swab (methicillin-resistant Staphylococcus aureus, MRSA). A clinical diagnosis of tympanic membrane perforation was made and the patient was treated with an innovative surgery approach: type 1 Tympanoplasty with the application of platelet gel. 


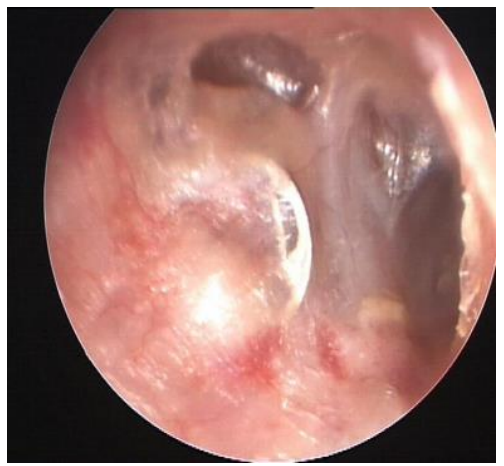

Figure 1: Clinical examination revealed single, inferior and marginal, "kidney shape" tympanic membrane perforation, with mild reddened and exuding margins, surrounded by an area of miringitis

\section{Case Presentation and Method}

Platelet gel was obtained by extracting $45 \mathrm{ml}$ of autologous peripheral blood, which was then immediately processed at room temperature in a dedicated device Angel System. Whole blood underwent two consecutive centrifugations as standard protocol (hematocrit 7\%) to produce $2 \mathrm{ml}$ of platelet-rich plasma (Mann et al., 2001). The thrombin fraction was then produced from platelet-poor plasma with a specific kit that enables the extraction of autologous thrombin from the platelet rich plasma (PRP): when added to the harvested PRP, thrombin activates the coagulation cascade, induces the platelet's degranulation and initiates the formation of the fibrin matrix (I-Kung et al., 2016; Zhang et al., 2019). After this first phase that was done by physician experienced in Transfusion Medicine, the Otorhinolaryngogyst has placed a cotton soaked in anesthetic on tympanic membrane and into the external auditory canal for a time of 30 minutes. Then, he has dipped a modified haemostatic gelatin (Gelita®, Braun, Melsungen, Germany) into the PRP for 2 minutes, and then placed it into tympanic cavity, applying a membrane of bovine collagen (Tutopatch®, Tutogen Medical, Bavaria, Germany). The cotton was removed on the fifth post-operative day.

\section{Discussion}

As far as we know there are no similar studies that evaluate this surgical approach with autologous platelet gel in association with a membrane of collagen. The bioactive molecules secreted from PRP facilitate the ability of leukocytes to clear pathogens and necrotic tissue, which promotes wound healing. As demonstrated in this report. We have applied a conventional surgical technique as type 1 miryngoplasty with a bioactive graft to induce wound healing in a soft tissue as tympanic membrane.

At 5 months follow-up, the perforation treated with this new approach was closed, without any morbidity (Fig. 2) and the audiometric exam showed a little improvement of deafness (Fig. 3). 


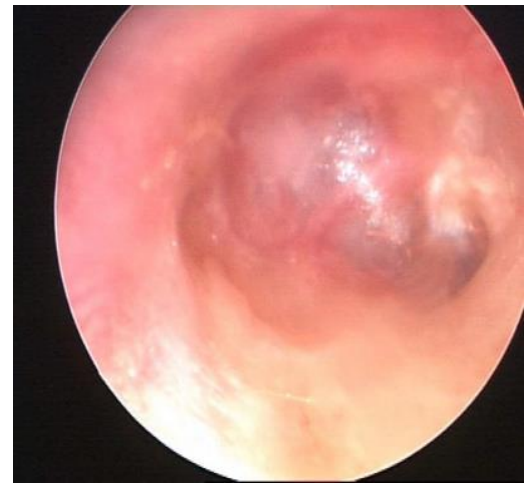

Figure 2: At 5 months follow-up, the perforation treated with this new approach was closed, without any morbidity
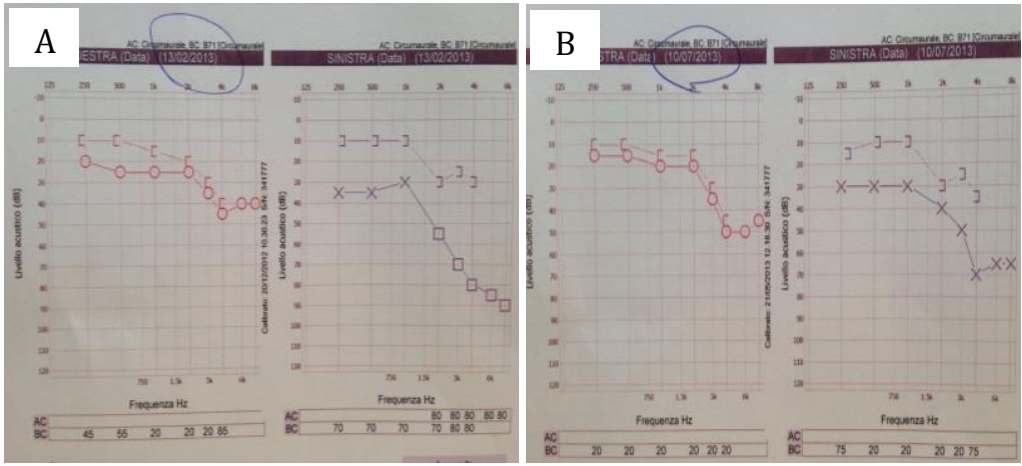

Figure 3: Pre (A) and post-tympanoplasty (B) type 1 (platelet gel approach) audiometric exam

\section{Conclusions}

In conclusion, this surgical procedure of type 1 tympanoplasty with platelet gel is a non-invasive and safe approach for resolving a situation of tympanic perforation. Furthermore, we report faster recovery for the patient without any complication and with a little improvement of deafness (see audiometric exam).

\section{Conflict of Interest}

The Authors declare no conflict of interest and no financial interest.

\section{References}

Adler SC and Kent KJ. Enhancing healing with growth factors. Facial Plast Surg Clin North Am 2002; 10: 129-146.

Bhanot S and Alex JC. Current applications of platelet gels in facial plastic surgery. Facial Plast Surg 2002;18: 27-33.

I-Kung Wu P, Diaz P, Borg-Stein J. Platelet-Rich Plasma. Phys Med Clin N Am 2016; 27: 825-853. 
Mann D, Plosker H, Winland-Brown JE. The use of autologous platelet rich plasma (platelet gel) and autologous platelet poor plasma (fibrin glue) in cosmetic surgery. Plast Reconstr Surg 2001; 107: 229-237.

Pierce GF, Mustoe TA, Altrock BW, Deuel TF, Thomason A. Role of platelet derived growth factor in wound healing. J Cell Biochem 1991; 45: 319-326.

Zhang W, Guo Y, Kuss M, Shi W, Aldrich AL, Untrauer J, Kielian T, Duan B. Platelet-Rich Plasma for the Treatment of Tissue Infection: Preparation and Clinical Evaluation. Tissue Eng Part B Rev 2019; 25 : 225-236. 